\title{
What is the real level of physical disability among elderly Chinese? A comparative study based on four national social surveys
}

Xiaochun Qiao ( $\nabla$ qxchcn@aliyun.com )

Peking University https://orcid.org/0000-0003-1767-440X

He Chen

Renmin University of China

Yan Liu

Peking University

Xiaolan Wu

China Research Center on Aging

Research article

Keywords: activities of daily living, the prevalence of physical disability, comparative study, China

Posted Date: December 26th, 2019

DOI: https://doi.org/10.21203/rs.2.19632/v1

License: (c) (1) This work is licensed under a Creative Commons Attribution 4.0 International License.

Read Full License 


\section{Abstract}

\section{Background}

Along with the increasing availability of national surveys on elderly health, the real level of physical disability among elderly Chinese is still a myth due to the data incomparability. This study aims to add knowledge on this topic by improving data comparability across surveys.

\section{Methods}

Data were drawn from four national surveys, including Chinese Longitudinal Healthy Longevity Survey (CLHLS) 2014, Sample Survey of the Aged Population in Urban/Rural China (SSAPUR) 2015, China Health and Retirement Longitudinal Study (CHARLS) 2015, and China Longitudinal Aging Social Survey (CLASS) 2014. The study subjects were people aged 65 years or above living in communities. The final sample size in four surveys analyzed was between 5008 in CHARLS and 144.0 thousand in SSAPUR. Katz activities of daily living (ADL) index was used to assess physical functioning. A series of measures were taken to harmonize $A D L$ index across surveys. The reliability and validity of index was examined with Cronbach's alpha, confirmatory and exploratory factor analysis. A standardized physical disability prevalence was computed by applying sex and age structure in 2015 China national $1 \%$ sample survey to the crude prevalence.

Results

All the Katz ADL indexes in four surveys had good internal consistency with Cronbach's alpha higher than 0.80 and good construct validity according to confirmatory and exploratory factor analysis results. After standardization, the physical disability prevalence remained significantly different across surveys, ranging from $10.21 \%(9.47 \%-10.95 \%)$ to $18.07 \%$ (17.88\%-18.27\%). The total number of older population with physical disability in China was between 14.68 million and 25.98 million, which was a wide range. The variation in physical disability prevalence was higher with respect to older age, rural residence, and mild disability.

\section{Conclusions}

Considering all the surveys have both advantages and disadvantages in assessing ADL disability, it's difficult to conclude the best data source on this topic. We suggest that future studies on, such as elderly physical disability and long-term care need, use multiple data sources at the same time to produce robust results.

\section{Background}

In 2018 , about 166.58 million people aged 65 years or above in China, accounting for $11.9 \%$ of total population in the country [1] and over $20 \%$ of elderly population in the world [2]. China, as a country with Confucian culture and rapid demographic transition, is confronted with a great challenge for aged care [3, 
4]. To build a sustainable and efficient aged care system, it's critical to assess the physical functioning of older population [3]. The index of independence in activities of daily living (ADL) developed by Katz and colleagues is one of the most widely used tool to serve this purpose [5-8]. The Chinese national surveys incorporating $A D L$ and relevant literatures are more and more available in recent years [9-14]; however, the real level of physical disability among older people in China is still a myth.

The data comparability across surveys brings new issues in assessing elderly physical disability in China. Back to early 1990s, there was no national survey focused on elderly health in this country. Older population is covered in the surveys on adults or general population, such as China Health and Nutrition Survey [15]. It usually results in a small number of older respondents and probably insufficient and unstable estimation of physical disability prevalence, especially in the oldest-old [16, 17]. At the turn of century, this situation get improved significantly with the implementation of Chinese Longitudinal Healthy Longevity Survey (CLHLS) in 1998 and Sample Survey of the Aged Population in Urban/Rural China (SSAPUR) in 2000 [13, 18, 19]; about a decade later, the implementation of China Health and Retirement Longitudinal Study (CHARLS) in 2011 [20] and China Longitudinal Aging Social Survey (CLASS) in 2014 [14] further provide abundant data for researchers. All of them are national surveys focused on elderly population and have good data quality $[13,14,19,20]$, whereas their survey designs are different. For example, the sample sizes range from 7192 in CLHLS 2014 to 224.1 thousand in SSAPUR 2015; CLHLS employs a targeted randomly sampling technique and the others probability sampling. The ADL index is included in the four surveys with differences in item setting. For example, CHARLS defined those who can use a catheter (conduit) or pouch by themselves as having no difficulty with urinary continence, while CLASS regards these people as disabled. These differences in survey design and ADL index potentially bring incomparability in assessing elderly physical functioning across data.

Despite the critical role of physical disability prevalence in monitoring elderly health and developing aged care policy, such incomparability in ADL measurement is largely overlooked by existing literatures, which leads to varying results in physical disability assessment [9-11, 21-23]. The physical disability prevalence for older people aged 60 years or above was 10.14\% in CHARLS 2015 [23], 9.3\% in CLASS 2014 [14], 4.1\% in SSAPUR 2015 [13], and 17.43\% in CLHLS 2014 (for those aged 65 years or above) [24]. Based on our comprehensive literature review, only one Chinese paper has so far discussed this issue [25]. Zhang and Wei found, after harmonizing the ADL index, the disparities in physical disability prevalence still exist, i.e., 10.48\% in CLHLS 2011, 11.25\% in CHARLS 2011, and 13.31\% in SSAPUR 2010 [25]. This study didn't perform reliability and validity assessment on ADL index. With the new launched survey of CLASS and new waves of CLHLS, CHARLS, and SSAPUR, an updated assessment on physical disability prevalence among older people in China is needed.

Therefore, based on data from four national surveys including CLHLS 2014, SSAPUR 2015, CHARLS 2015, and CLASS 2014, this study aimed to: (1) compare and harmonize the ADL index used in different surveys to increase the data comparability; (2) employ Cronbach's alpha to evaluate the internal consistency and confirmatory and exploratory factor analysis to evaluate the construct validity of ADL index; (3) analyze the prevalence of physical disability of older people across surveys. 


\section{Methods}

\subsection{Data}

Data were drawn from four national social surveys, namely CLHLS 2014, SSAPUR 2015, CHARLS 2015, and CLASS 2014. Their basic information is presented in Table 1. CLHLS has the longest history dating back to 1998 , followed by SSAPUR, CHARLS and CLASS. All the surveys adopted a complex survey design to achieve a better national representativeness. Their data quality has been verified in previous literatures $[13,14,19,20]$. These data had been widely used in the studies on elderly health in China [911]. The detailed information on the survey design is described in Additional file 1. As CLHLS mainly surveyed population aged 65 years or above, this study focused on this age group in all the four datasets. The final sample size varied from 5008 in CHARLS 2015 to 144.0 thousand in SSAPUR 2015.

Table 1 Survey general information

\begin{tabular}{|c|c|c|c|c|c|}
\hline \multirow[t]{2}{*}{ Survey } & \multirow[t]{2}{*}{ Survey year } & \multirow[t]{2}{*}{ Sample design } & \multicolumn{3}{|c|}{ Sample size in recent wave } \\
\hline & & & Total & $\begin{array}{l}\text { Total } \\
65+\end{array}$ & $\begin{array}{l}\text { Final } \\
\text { sample } \\
\text { analyzed } \\
65+\end{array}$ \\
\hline CLHLS & $\begin{array}{l}\text { 1998, 2000, 2002, 2005, } \\
\text { 2008-2009, 2011-2012, } 2014\end{array}$ & targeted randomly sampling & 7192 & 7107 & 6498 \\
\hline SSAPUR & 2000, 2006, 2010, 2015 & $\begin{array}{l}\text { multistage, stratified, probability } \\
\text { proportional to size random sampling }\end{array}$ & 224142 & 154143 & 143990 \\
\hline CHARLS & $2011,2013,2015$ & $\begin{array}{l}\text { multistage, stratified, probability } \\
\text { proportional to size random sampling }\end{array}$ & 21789 & 6519 & 5008 \\
\hline CLASS & 2014 & $\begin{array}{l}\text { stratified, multistage probability } \\
\text { sampling }\end{array}$ & 11511 & 8439 & 7858 \\
\hline
\end{tabular}

Note: PPS, probability proportional to size random sampling method.

\subsection{Measurement}

Physical functional ability was measured with ADL index proposed by Katz and colleagues $[5,6]$, which includes six activities, namely bathing, dressing, using the toilet, transferring from bed to chair, continence, and feeding. The four surveys asked ADL items in slightly different ways (Additional file 2). To assess the physical functioning of elderly respondents as comparably as possible, we did the following to harmonize the ADL index. For each item in CLHLS, CHARLS and CLASS, we combined the last two options to indicate that respondents are partly or completely dependent on the item and require long-term care. The situation for SSAPUR was more complicated. In this survey, the second option for each item (i.e., Somewhat difficult) does not clarify whether a help is required to finish the activity. Thus, we analyzed the data in two ways, one combining the last two options (SSAPUR-1) and the other only using the last option (SSAPUR-2) to indicate dependency in activities. The physical disability prevalence obtained in SSAPUR-1 is expected to be higher than that in SSAPUR-2. Furthermore, CLASS split continence into two questions, fecal and urinary continence. CLASS respondents were defined as dependent in continence as long as they reported "Usually" to any of the two items. In SSAPUR, the 
question on continence had three options "Fecal incontinence", "Urinary incontinence", and "None". SSAPUR respondents who chose any of the first two options were regarded as dependent in continence. Respondents who were dependent in any of the six activities were regarded as having physical disability. Those with 1 to 2 activities disabled were regarded as having mild physical disability, 3 to 4 moderate physical disability, and 5 to 6 severe physical disability.

Other variables were used to analyze the distribution of respondents, including five-years age groups (6569 years to 90 years or above), sex (male and female), residence (urban and rural), education (no school, primary school, high school and above, and don't know or missing) and whether being married (yes and no).

\subsection{Analyses}

Frequencies and proportions were used to describe the basic distribution of categorical variables; mean and standard deviation were used for continuous variables. T-test was used to examine the significance in difference in proportion of physical disability.

Internal consistency and construct validity of ADL index in four surveys were assessed before further analysis. Firstly, a value of Cronbach's alpha higher than 0.70 was regarded as acceptable internal consistency [26]. Secondly, we used confirmatory and exploratory factor analyses to assess construct validity. In confirmatory factor analyses, we set the number of factor to be one. If the model fit satisfies the following criteria, the six $A D L$ items are regarded to have unidimensional construct, i.e., $/ \mathrm{df}<3.0$, comparative fit index $(\mathrm{CFI}) \geq 0.95$, Tucker-Lewis coefficient $(T L I) \geq 0.95$, and root mean square error of approximation (RMSEA) $\leq 0.05$ [27]. Otherwise, exploratory factor analysis is performed to examine construct validity. Kaiser-Meyer-Olkin (KMO) was calculated to measure the sampling adequacy of the data. The significance of Barlett test was examined. The principal component method was performed to extract the corresponding factors. The number of factors was determined by their eigenvalues greater than 1 . The cumulative variance was estimated to demonstrate the variance explained by the factors. Items with factor loading higher than 0.40 were retained. As the six ADL items are binary, we firstly computed tetrachoric correlation coefficient matrix and then run confirmatory and exploratory factor analyses using the matrix [28]. Moreover, as it's difficult to analyze SSAPUR data due to its large sample size, we resampled $25 \%$ of respondents ( 36,350 cases) to perform factor analyses; the resampled were randomly drawn following a consistent distribution of sex, age and residence with the whole sample. A total of six resamples of SSAPUR were obtained and the analyses results were stable across resamples. We only presented results from one of such resamples to be concise.

Considering the crude physical disability prevalence was affected by the sex and age structure in each dataset, we computed standardized physical disability prevalence by applying sex and age structure in 2015 China national $1 \%$ sample survey to the crude physical disability prevalence. We further estimated the number of elderly people with physical disability through multiplying sex-age-specific prevalence of physical disability by sex-age-specific number of elderly population in China. 
The analyses were weighted and performed with Stata MP (version 15.0) for CLHLS 2014, CHARLS 2015, and CLASS 2014, and PASW (version 18) for SSAPUR 2015.

\section{Results}

\subsection{Sample characteristics}

Table 2 shows the characteristics of respondents aged 65 or above in four surveys. SSAPUR, CHARLS, and CLASS had similar age structures and CLHLS had an older age structure than other surveys with those aged $65-69$ years accounting for only $26.70 \%$ and 80 years and above $23.18 \%$. The proportion of male was $45 \%-49 \%$ in four surveys. The proportion of urban respondents was over $50 \%$ in both SSAPUR and CLASS, while the corresponding figures were $44.03 \%$ in CLHLS and $31.05 \%$ in CHARLS. Those without any education accounted for about $1 / 3$ in SSAPUR and CLASS and nearly $40 \%$ in the other surveys; those with primary education accounted for $32.28 \%$ in CLASS to around $42 \%$ in other surveys. The proportion ever attending high school and above ranged from $16.24 \%$ in CHARLS to almost $1 / 3$ in CLASS. The proportion of married people was $62 \%-68 \%$ in four surveys.

Table 2 Characteristics of respondents aged 65 or above in four surveys (\%)

\begin{tabular}{lcccc}
\hline & CLHLS & SSAPUR & CHARLS & CLASS \\
\hline $\mathrm{N}$ & 6498 & 143990 & 5008 & 7858 \\
Age groups & & & & \\
$65-69$ & 26.70 & 34.94 & 35.51 & 33.54 \\
$70-74$ & 28.76 & 24.69 & 26.95 & 25.55 \\
$75-79$ & 21.35 & 19.34 & 18.71 & 20.96 \\
$80-84$ & 14.08 & 12.95 & 11.52 & 13.18 \\
$85-89$ & 6.43 & 6.04 & 5.12 & 5.12 \\
90 and above & 2.67 & 2.06 & 2.19 & 1.66 \\
Sex & & & & \\
Male & 48.39 & 47.22 & 44.82 & 48.81 \\
Female & 51.61 & 52.78 & 55.18 & 51.19 \\
Residence & & & & \\
Urban & 44.03 & 51.92 & 31.05 & 53.19 \\
Rural & 55.97 & 48.08 & 68.95 & 46.81 \\
Education & & & & \\
$\quad$ No school & 38.29 & 33.72 & 39.51 & 33.29 \\
Primary school & 42.57 & 41.24 & 41.27 & 34.39 \\
High school and above & 18.82 & 24.72 & 16.24 & 32.28 \\
Don't know or missing & 0.32 & 0.32 & 2.98 & 0.05 \\
Married & & & & \\
$\quad$ Yes & 62.31 & 62.49 & 66.38 & 68.49 \\
No & 36.52 & 33.76 & 33.61 & 31.40 \\
missing & 1.17 & 3.75 & 0.00 & 0.11 \\
\hline
\end{tabular}

Note: All the results are weighted. Proportions may not sum to $100.00 \%$ because of missing data.

\subsection{Reliability and validity assessment}


All the ADL indexes in four surveys had good internal consistency with Cronbach's alpha higher than 0.80 (Table 3). The model fit of confirmatory factor analyses satisfied all the criteria except $X_{2} / \mathrm{df}$ (Table 4 ).

We further performed exploratory factor analysis to examine the unidimensionality of the ADL index. The first factor in all the data had eigenvalues greater than 4.8 and could explain more than $87 \%$ of variance (Table 5). The factor loadings of items were higher than 0.65 (Table 6). The results show that ADL index in each survey had acceptable unidimensionality.

Table 3 The Cronbach's Alpha results in four surveys

\begin{tabular}{lc}
\hline Source & Cronbach's Alpha \\
\hline CLHLS & 0.9024 \\
SSAPUR-1 & 0.8650 \\
SSAPUR-2 & 0.8230 \\
CHARLS & 0.8189 \\
CLASS & 0.8535 \\
\hline
\end{tabular}

Table 4 Model fit of confirmatory factor analyses in four surveys

\begin{tabular}{lcccc}
\hline Source & $/ \mathrm{df}$ & CFI & TLI & RMSEA (90\%CI) \\
\hline CLHLS & 6.76 & 1.000 & 0.999 & $0.030(0.012,0.053)$ \\
SSAPUR-1 & 10.59 & 1.000 & 1.000 & $0.011(0.005,0.018)$ \\
SSAPUR-2 & 53.21 & 1.000 & 1.000 & $0.021(0.017,0.027)$ \\
CHARLS & 30.23 & 0.999 & 0.998 & $0.032(0.021,0.043)$ \\
CLASS & 16.16 & 1.000 & 0.999 & $0.030(0.018,0.044)$ \\
\hline
\end{tabular}

Note: CFI represents comparative fit index, TLI represents Tucker-Lewis coefficient, RMSEA represents root mean square error of approximation.

Table 5 The eigenvalues and cumulative proportion of the variance explained by the first factor in exploratory factor analyses

\begin{tabular}{lcc}
\hline Source & Eigenvalues & $\begin{array}{c}\text { Cumulative proportion of the variance } \\
\text { explained by factor (\%) }\end{array}$ \\
\hline CLHLS & 5.42 & 99.25 \\
SSAPUR-1 & 5.26 & 87.65 \\
SSAPUR-2 & 5.47 & 91.24 \\
CHARLS & 4.87 & 100.02 \\
CLASS & 5.08 & 98.85 \\
\hline
\end{tabular}

Table 6 Factor loadings of ADL items in four surveys in exploratory factor analyses

\begin{tabular}{lccccc}
\hline Items & CLHLS & SSAPUR-1 & SSAPUR-2 & CHARLS & CLASS \\
\hline Bathing & 0.94 & 0.96 & 0.97 & 0.90 & 0.94 \\
Dressing & 0.99 & 0.99 & 1.00 & 0.93 & 0.97 \\
Using the toilet & 0.99 & 0.98 & 0.99 & 0.88 & 0.98 \\
Continence & 0.82 & 0.66 & 0.73 & 0.81 & 0.71 \\
Transferring & 0.98 & 0.98 & 1.00 & 0.96 & 0.94 \\
Feeding & 0.97 & 0.95 & 0.97 & 0.91 & 0.95 \\
\hline
\end{tabular}




\subsection{Prevalence of physical disability}

The crude prevalence of physical disability in elderly people significantly varied, from $11.06 \%(95 \% \mathrm{Cl}$, $10.29 \%-11.83 \%$ ) in CLHLS to $18.85 \%$ (18.65\%-19.05\%) in SSAPUR-1 (Table 7). Its mean was $14.92 \%$ among surveys. The sex-age-standardized prevalence of physical disability was slightly lower than the crude, but the variation still existed, with prevalence ranging from $10.21 \%(9.47 \%-10.95 \%)$ in CLHLS to $18.07 \%$ (17.88\%-18.27\%) in SSAPUR-1 and a mean of 14.34\%. This suggests that the disparities in physical disability prevalence across surveys were not a result from respondents' sex and age structure.

Table 7 The prevalence of physical disability among people aged 65 years or above in four surveys (\%)

\begin{tabular}{lccccc}
\hline & \multicolumn{2}{c}{ Crude prevalence } & & \multicolumn{2}{c}{ Standardized prevalence } \\
\cline { 2 - 3 } \cline { 5 - 6 } Source & Estimate & $95 \%$ CI & & Estimate & $95 \%$ CI \\
\hline CLHLS & 11.06 & $10.29-11.83$ & & 10.21 & $9.47-10.95$ \\
SSAPUR-1 & 18.85 & $18.65-19.05$ & & 18.07 & $17.88-18.27$ \\
SSAPUR-2 & 12.47 & $12.30-12.64$ & & 11.99 & $11.83-12.16$ \\
CHARLS & 16.27 & $16.26-16.28$ & & 16.04 & $16.03-16.05$ \\
CLASS & 15.94 & $15.13-16.75$ & & 15.39 & $14.60-16.19$ \\
\hline
\end{tabular}

Note: Standardized physical disability prevalence is obtained by applying sex and age structure in 2015 national $1 \%$ sample survey in China to the crude physical disability prevalence.

As shown in Table 8, the total number of older population with physical disability in China was 14.68 million to 25.98 million. The number of older population with severe physical disability, which Chinese long-term care insurance pilots focus on, was 2.09 million to 5.10 million.

Table 8 The number of people aged 65 years or above with physical disabilities by severity (millions)

\begin{tabular}{lcccc}
\hline Source & Mild disability & Moderate disability & Severe disability & Total \\
\hline CLHLS & 8.77 & 2.37 & 3.52 & 14.68 \\
SSAPUR-1 & 16.76 & 4.12 & 5.10 & 25.98 \\
SSAPUR-2 & 13.89 & 1.25 & 2.09 & 17.24 \\
CHARLS & 16.30 & 3.84 & 2.91 & 23.05 \\
CLASS & 14.76 & 3.16 & 4.22 & 22.13 \\
\hline
\end{tabular}

Note: Due to rounding, the sum of mild, moderate, and severe physical disability isn't equal to the total.

To look deeper into the factors behind these disparities, we further analyzed the detailed distribution of physical disability prevalence by age, sex, residence, and severity of physical disability. Figure 1 shows that, in most sex and age groups examined, physical disability prevalence in CLHLS was the lowest, followed by SSAPUR-2. In groups aged 65 to 84 years, physical disability prevalence was the highest in SSAPUR-1. In groups aged 90 years or above, the prevalence in SSPAUR- 1 and CLASS were similar, around $52 \%$ in male and $60 \%$ in female; among CHARLS respondents, the physical disability prevalence in this age group was $59 \%$ in male, much higher than that in female (39\%), which is unexpected. Generally, the range in physical disability prevalence enlarged along with age. 
Figure 2 shows that the disparities in physical disability prevalence in rural older people were much higher than that in their counterparts. In urban area, the prevalence of physical disability in SSAPUR-2 and CLHLS was the lowest, and that in SSPAUR-1 and CLASS was the highest. The corresponding prevalence in CHARLS was more fluctuant with age than other surveys. For example, the physical disability prevalence in age group 80-84 in CHARLS was $12.47 \%$, much lower than that in younger age group 75-79 (15.70\%). In rural area, physical disability prevalence was the lowest in CLHLS, followed by that in SSAPUR-2. In rural respondents aged 90 or above, the prevalence was lowest in CHARLS and CLHLS (31\%) and highest in CLASS (69\%).

Figure 3 shows the disparities in physical disability prevalence across severity. For mild physical disability, the prevalence was the lowest in CLHLS. Different from other surveys, the prevalence of mild physical disability in CHARLS went sharply down in group aged 90 years or above, which may be related to the small sample size in this age group. For moderate and severe physical disability, the prevalence was the lowest in SSAPUR-2 and CLHLS and highest in SSAPUR-1.

\section{Discussion}

Based on four national social surveys on elderly population in China, CLHLS, SSAPUR, CHARLS, and CLASS, this paper compared and harmonized the Katz ADL index, assessed their reliability and validity, and analyzed prevalence of physical disability. Its main merit is to avoid the bias in estimation of physical disability prevalence based on one single data source, which is critical to understand the real level of physical disability and develop aged care policies like the on-going long-term care insurance pilots in China.

The physical disability prevalence estimated using data from four surveys significantly vary, even after we used the survey data in most similar years, harmonized the ADL index, and standardized the crude physical disability prevalence using national sex and age structure. Such results are consistent with previous studies $[9,11,13,23,25]$. The factors contribute to the disparities in physical disability prevalence across surveys at least include the following aspects. Firstly, difference exists in the design of the four social surveys. CLHLS adopts a targeted random-sample method and is not nationally representative [11], while other three surveys are designed to be representative of elderly population in China. Secondly, despite our best effort, differences still exist in the ADL index across four surveys. Unlike in other three surveys, the second options in CLHLS for eating, dressing, toileting, getting in and out of bed, and bathing are "some difficulties", from which we can't tell if a help from others is needed to perform these tasks. To cope with this, we analyzed two situations, SSAPUR-1 and SSAPUR-2 regarding those choosing "some difficulties" as disabled and nondisabled with these tasks, respectively. The physical disability prevalence based on SSAPUR-1 (18.07\%) is expected to overestimate the real level of physical disability and SSAPUR-2 underestimate (11.99\%). A long-standing duration of the ability restriction, "three months", is required in CHARLS. CLHLS specify "at least the last six months" in a question before ADL index, but not explicitly indicate the duration in the following ADL index. Both CLASS and SSAPUR did not have any time limitation, which may result in overestimation of physical disability 
prevalence [7]. The order and description of ADL items also vary across surveys, whereas it's difficult to determine their potential impact on physical disability prevalence estimation.

The real level of physical disability prevalence among elderly Chinese might be between $10.21-18.07 \%$. All the four social surveys, i.e. CLHLS, CLASS, CHARLS and SSAPUR, have good data quality $[13,14,19$, $20]$ and the ADL index in four surveys passed through reliability and validity examination. On the other hand, all of the four surveys have some disadvantages in measuring ADL disability. CLHLS is not nationally representative, SSAPUR and CLASS don't set a time restriction for the ability assessment, and CHARLS has a small size of oldest-old and urban residents. Considering these above factors, we can't conclude any of the survey data better than others in estimating physical disability prevalence. We also found that the variation in physical disability prevalence was higher with respect to older age, rural residence, and mild disability.

Last but not least, researchers should be careful in using CLHLS, SSAPUR, CHARLS, and CLASS in providing evidence for aged care policies. Firstly, considering the disparities in physical disability prevalence in these four social surveys, analysis using multiple data sources probably produce more robust results than that using single data source. For example, our results show that the number of severely disabled older population is between 2.09 million to 5.10 million in China, which is still a wide range for long-term care policies. Any recommendation based on single data source may introduce bias into policymaking. Secondly, the physical disability assessment tools are different between social surveys and long-term care insurance practices in China. Katz ADL is used in all the four social surveys, and the Barthel index is used in most long-term care insurance pilots [29]. These two tools probably produce different physical functional assessment results [7, 30]. Additionally, at least six months of physical disability is also required for eligibility of long-term care insurance benefits. However, in CLASS and SSAPUR, the duration of ability restriction is not indicated in the questionnaire, which may include some short-term physical disability and overestimate the needs for long-term care. In the future, social surveys could consider including physical disability assessment tool used in long-term care insurance into the questionnaire, which will increase the implication for policy practices.

This study has some limitations. Firstly, despite our best effort, the physical disability prevalence measured with ADL index in the four social surveys is not completely comparable. This is because we aren't able to change the survey designs and questionnaire setting after the data had been collected. Secondly, as required to be stored in China Research Center on Ageing, the SSAPUR data was analyzed in a slightly different way with other data source. We used statistical software PASW (version 18) to analyze the SSAPUR data and Stata MP (version 15.0) for other three sources. Six resamples of 25\% SSAPUR respondents were drawn to perform the factor analysis due to the large sample size of SSAPUR and the limited computing capability of computers. We estimated the tetrachoric correlation matrix in PASW (version 18) according to Lorenzo-Seva and Ferrando [31] and using "tetrachoric" module in Stata MP (version 15.0). Considering the consistence between the two statistical softwares and stable results across SSAPUR resamples, the bias introduced by the different analysis approaches could be neglected. 
After we improved the comparability by drawing data from the surveys in most similar years, harmonizing the Katz ADL index, and standardizing the crude physical disability prevalence, the prevalence of physical disability among elderly people still significantly differed across four national social surveys in China, ranging from $10.21 \%$ (9.47\%-10.95\%) in CLHLS to $18.07 \%$ (17.88\%-18.27\%) in SSAPUR-1. All the indexes had satisfying reliability and validity assessment results and surveys had good data quality, whereas all the surveys had some disadvantages in measuring physical disability. Therefore, it's difficult to conclude the best data source to estimate physical disability prevalence. We suggest that future studies on, such as elderly physical disability and long-term care need, use multiple data sources at the same time to produce robust results. Considering that China has the largest number of elderly people in the World, this study may shed some lights on assessing global elderly disability. Furthermore, researchers in other countries also need to pay attention on the potential differences in physical disability prevalence estimated from different data sources.

\section{Abbreviations}

ADLs: Activities of Daily Living

CLHLS: Chinese Longitudinal Healthy Longevity Survey

SSAPUR: Sample Survey of the Aged Population in Urban/Rural China

CHARLS: China Health and Retirement Longitudinal Study

CLASS: China Longitudinal Aging Social Survey

CFI: comparative fit index

TLI: Tucker-Lewis coefficient

RMSEA: root mean square error of approximation

KMO: Kaiser-Meyer-Olkin

\section{Declarations}

\section{Acknowledgements}

Not Applicable.

\section{Funding}

This study was funded by Major Program of National Fund of Philosophy and Social Science of China (CN) (No. 17ZDA124), Programme grants from Data Center for Management Science, NSFC-PKU (No. 2018KEY05), and China Ministry of Education Humanities and Social Science Research Youth Fund 
Project (No. 18YJC840003). The funding bodies had no role in study design, data collection, analysis, interpretation, or writing of the article. The authors had full access to all the data in the study and final responsibility for the decision to submit for publication. The authors were independent from the study sponsors.

\section{Availability of data and materials}

The secondary data CLHLS 2014, SSAPUR 2015, CHARLS 2015, and CLASS 2014 have been used in this study. The former three datasets is freely available in their official website: CLHLS 2014 (https://sites.duke.edu/centerforaging/programs/chinese-longitudinal-healthy-longevity-survey-clhls/), CHARLS 2015 (http://charls.pku.edu.cn/), and CLASS 2014 (http://class.ruc.edu.cn/). SSAPUR 2015 data needs to be applied from China Research Center on Ageing.

\section{Authors' Contributions}

$H C, Y L, X L W$, and $X C Q$ conceived the study together. $H C$ and $Y L$ reviewed the literatures, analysed the data, and prepared the first draft. HC, YL, XLW, and XCQ revised the draft. The final version of manuscript was read and approved by all the four authors.

\section{Ethics approval and consent to participate}

The data used in our research is secondary data. All the data was anonymised before its use. No ethics approval or consent to participate is needed.

\section{Consent for publication}

Not applicable.

\section{Competing interest}

The authors declare that they have no competing interests.

\section{References}

1. National Bureau Statistics of China. 2018 National Economic and Social Development Statistics Report of China. http://www.stats.gov.cn/tjsj/zxfb/201902/t20190228_1651265.html; 2019.

2. United Nations DoEaSA, Population Division. World Population Prospects 2019, Online Edition edn: United Nations; 2019.

3. Glinskaya E, Feng Z (eds.). Options for Aged Care in China: Building an Efficient and Sustainable Aged Care System: World Bank Group; 2018.

4. Feng Z, Liu C, Guan X, Mor V. China's Rapidly Aging Population Creates Policy Challenges In Shaping A Viable Long-Term Care System. Health Aff. 2012;31(12):2764-2773. 
5. Katz S, Ford A, Moskowitz R, Jackson B, Jaffe M. Studies of illness in the aged. The index of ADL: a standardized measure of biological and psychosocial function. JAMA. 1963;185(12):914-919.

6. Katz S, Downs TD, Cash HR, Grotz RC. Progress in development of the index of ADL. Gerontologist. 1970;10(1):20-30.

7. McDowell I. Measuring Health: A Guide to Rating Scales and Questionnaires: Oxford University Press; 2006.

8. Gill TM. Assessment of function and disability in longitudinal studies. J Am Geriatr Soc. 2010;58 Suppl 2:308-312.

9. Zhang P-D, Lv Y-B, Li Z-H, Yin Z-X, Li F-R, Wang J-N, Zhang X-R, Zhou J-H, Wu X-B, Duan J et al. Age, period and cohort effects in activities of daily living, physical and cognitive functioning impairment among the oldest-old in China. The journals of gerontology Series A, Biological sciences and medical sciences. 2019. https://doi.org/10.1093/gerona/glz196.

10. Hou C, Ping Z, Yang K, Chen S, Liu X, Li H, Liu M, Ma Y, Van Halm-Lutterodt N, Tao L et al. Trends of Activities of Daily Living Disability Situation and Association with Chronic Conditions among Elderly Aged 80 Years and Over in China. Journal of Nutrition Health \& Aging. 2018;22(3):439-445.

11. Zeng Y, Feng Q, Hesketh T, Christensen K, Vaupel JW. Survival, disabilities in activities of daily living, and physical and cognitive functioning among the oldest-old in China: a cohort study. Lancet. 2017;389(10079):1619-1629.

12. Liang Y, Welmer A-K, Wang R, Song A, Fratiglioni L, Qiu C. Trends in Incidence of Disability in Activities of Daily Living in Chinese Older Adults: 1993-2006. Journal of the American Geriatrics Society. 2017;65(2):306-312.

13. Dang J, Wei Y, Liu N. Survey Report on the Living Conditions of the Elderly in Urban and Rural China (2018). Beijing: Social Sciences Academic Press (China); 2017 (Chinese).

14. National Survey Research Center at Renmin University of China: 2014 China Longitudinal Aging Social Survey Report. In: National Survey Research Center at Renmin University of China; 2014 (Chinese).

15. Popkin BM, Du S, Zhai F, Zhang B. Cohort Profile: The China Health and Nutrition Survey-monitoring and understanding socio-economic and health change in China, 1989-2011. International Journal of Epidemiology. 2009;39(6):1435-1440.

16. Liang Y, Welmer AK, Wang R, Song A, Fratiglioni L, Qiu C. Trends in incidence of disability in activities of daily living in Chinese older adults: 1993-2006. J Am Geriatr Soc. 2016;65(2):306-312.

17. Chen $H$, Chen $G$, Song $X$, Zheng $X$. The association between socio-economic status and elderly instrumental activities of daily life in China. Chinese Journal of Epidemiology. 2010;31(2):237-238 (Chinese).

18. Hvistendahl M. Can China age gracefully? A massive survey aims to find out. Science. 2013;341(6148):831-832.

19. Zeng Y, George L, Vaupel J, Goodkind D. Chinese longitudinal healthy longevity survey: A unique data resource and research opportunities. Gerontologist. 2004;44:194-195. 
20. Zhao Y, Hu Y, Smith JP, Strauss J, Yang G. Cohort profile: the China Health and Retirement Longitudinal Study (CHARLS). Int J Epidemiol. 2014;43(1):61-68.

21. Qian J, Chen Y, Rao K. Trends Analysis and Prediction on Disability of China Mainland Aging Population and Policy Suggestion. Chinese Journal of Health Statistics. 2012;29(1):6-9 (Chinese).

22. Yang Y. Characterising Long Term Care Needs among Chinese Older Adults with Cognitive Impairment or ADL Limitations. Journal of cross-cultural gerontology. 2019; DOI:10.1007/s10823019-09382-8.

23. Liu D, Dai T, Huang J. Demand and utilization of medical service among home-dwelling elderly with disability in China: 2011-2015. Chinese Journal of Public Health. 2018;34(5):687-689 (Chinese).

24. Wu $B$, Dong $H$, Wang $Y$, Zhang $X$. Discrimination of disability and its influence on health life expectancy in Chinese elderly population. Population Journal. 2019;41(1):101-112 (Chinese).

25. Zhang W, Wei M. Disability level of the Chinese elderly: comparison from multiple data sources. Population Research. 2015;39(3):34-47 (Chinese).

26. Chiu E-C, Lee Y, Lai K-Y, Kuo C-J, Lee S-C, Hsieh C-L. Construct Validity of the Chinese Version of the Activities of Daily Living Rating Scale III in Patients with Schizophrenia. Plos One. 2015;10(6). DOI: 10.1371/journal.pone.0130702.

27. TA B. Confirmatory factor analysis for applied research, 2nd ed edn. New York: Guilford Press; 2015.

28. Garin N, Koyanagi A, Chatterji S, Tyrovolas S, Olaya B, Leonardi M, Lara E, Koskinen S, TobiaszAdamczyk B, Ayuso-Mateos JL et al. Global multimorbidity patterns: a cross-sectional, populationbased, multi-country study. The Journals of Gerontology Series A: Biological Sciences and Medical Sciences. 2015;71(2):205-214.

29. Zhao Y, Huang C, Liu S, Ye M, Li J, Xie H. Research progress of disability assessment tools for longterm care insurance. Chinese Nursing Management. 2019;19(1):113-119 (Chinese).

30. Hartigan I. A comparative review of the Katz ADL and the Barthel Index in assessing the activities of daily living of older people. International journal of older people nursing. 2007;2(3):204-212.

31. Lorenzo-Seva U, Ferrando PJ. TETRA-COM: A comprehensive SPSS program for estimating the tetrachoric correlation. Behav Res Methods. 2012;44(4):1191-1196.

\section{Additional Files}

Additional file 1: Introduction of four national social surveys on elderly people in China. This file describes the basic information on Chinese Longitudinal Healthy Longevity Survey (CLHLS), Sample Survey of the Aged Population in Urban/Rural China (SSAPUR), China Health and Retirement Longitudinal Study (CHARLS), and China Longitudinal Aging Social Survey (CLASS). (DOCX 24kb)

Additional file 2: Katz ADL Measurement in the Four National Surveys on Elderly People in China. This file presents and compares the Katz ADL measurement in CLHLS, SSAPUR, CHARLS and CLASS. Table S1 


\section{Figures}
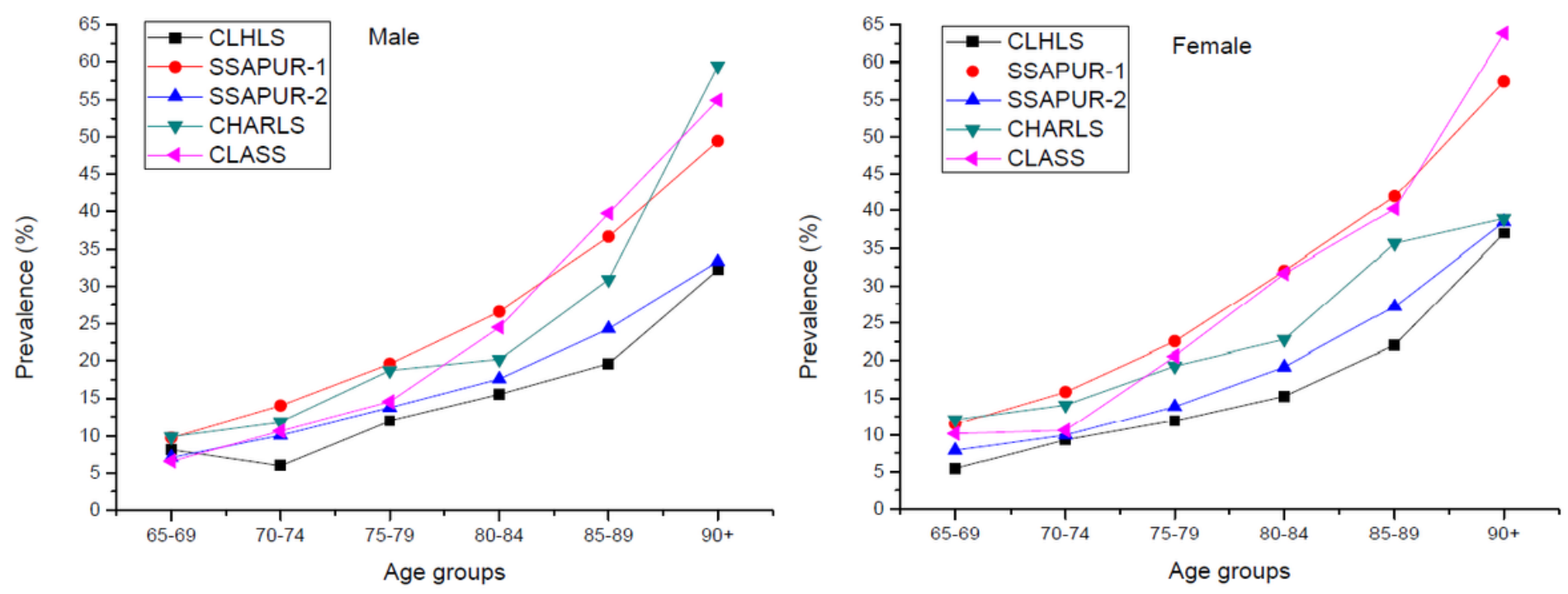

Figure 1

Physical disability prevalence among older people aged 65 years or above in four surveys by sex and age group (\%)
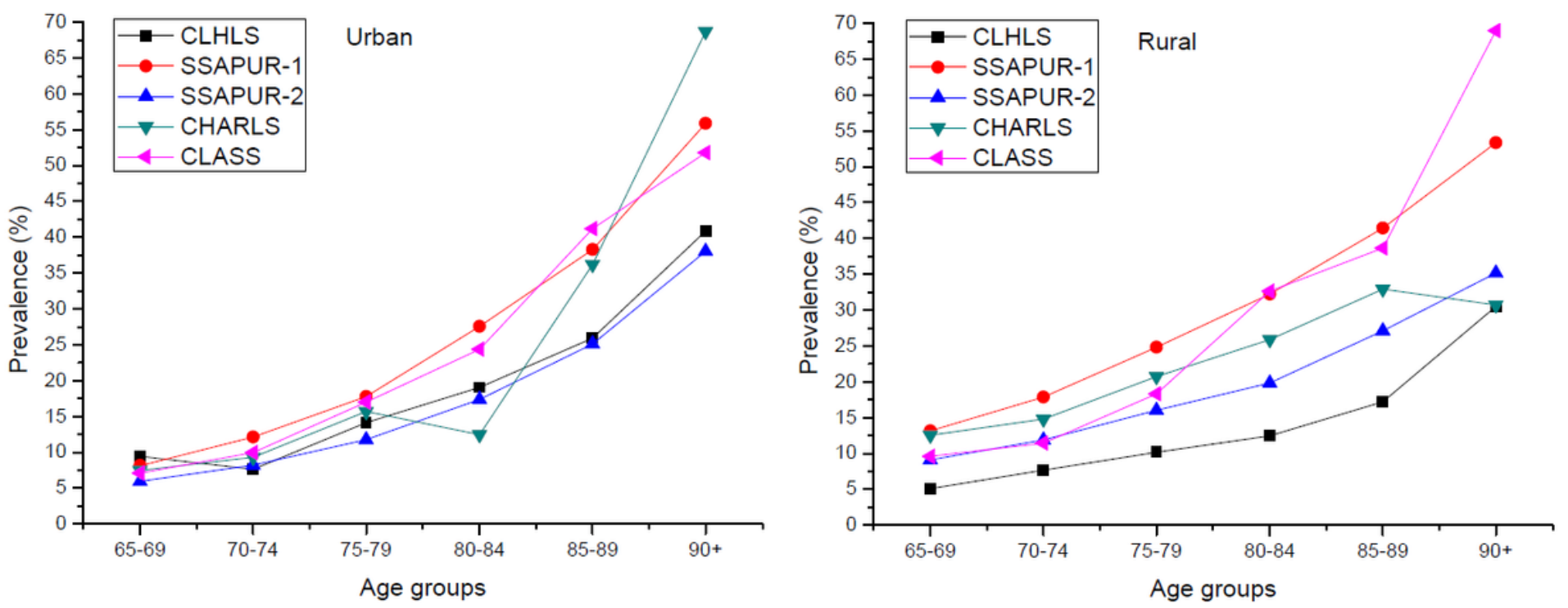

Figure 2

Physical disability prevalence among people aged 65 years or above in four surveys by residence and age group (\%) 

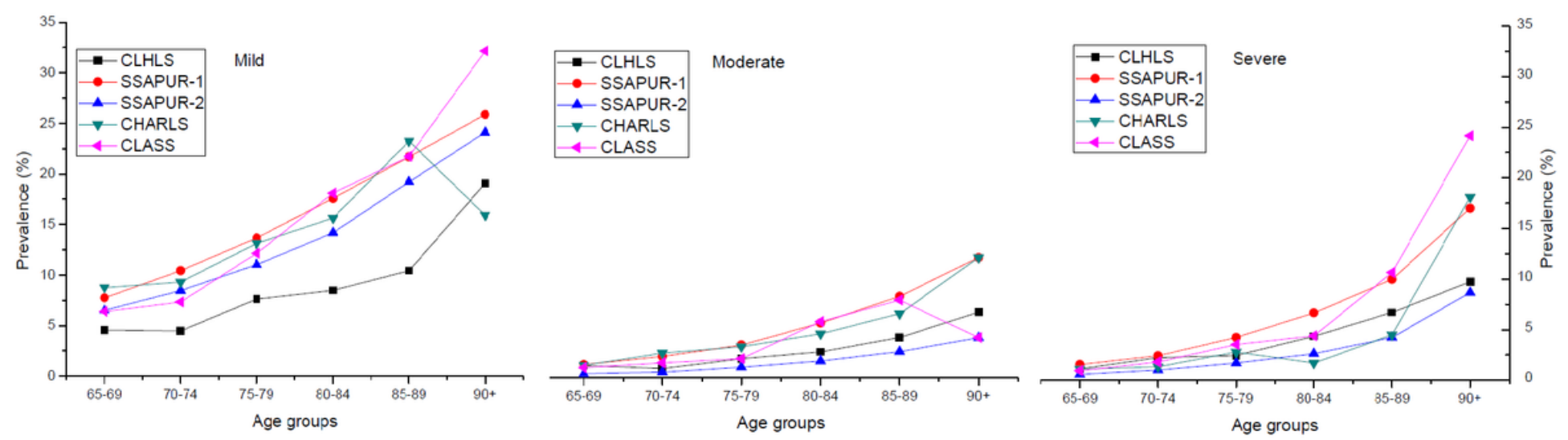

\section{Figure 3}

Physical disability prevalence among people aged 65 years or above in four surveys by severity and age groups (\%)

\section{Supplementary Files}

This is a list of supplementary files associated with this preprint. Click to download.

- Additionalfile2.docx

- Additionalfile1.docx 